April, 1997

UICHEP-TH/97-5

hep-th/9704119

\title{
Zero Curvature Formalism for Supersymmetric Integrable Hierarchies in Superspace
}

\author{
H. Aratynf ${ }^{a}$, A. Dasf b ${ }^{b}$ and C. Rasinariu ${ }^{a}$ \\ (a) Department of Physics \\ University of Illinois at Chicago \\ 845 W. Taylor St. \\ Chicago, IL 60607-7059 \\ (b) Department of Physics and Astronomy \\ University of Rochester \\ Rochester, N.Y. 14627
}

\begin{abstract}
We generalize the Drinfeld-Sokolov formalism of bosonic integrable hierarchies to superspace, in a way which systematically leads to the zero curvature formulation for the supersymmetric integrable systems starting from the Lax equation in superspace. We use the method of symmetric space as well as the non-Abelian gauge technique to obtain the supersymmetric integrable hierarchies of the AKNS type from the zero curvature condition in superspace with the graded algebras, $\operatorname{sl}(n+1, n)$, providing the Hermitian symmetric space structure.
\end{abstract}

\footnotetext{
${ }^{1}$ Work supported in part by U.S. Department of Energy, contract DE-FG02-84ER40173

${ }^{2}$ Work supported in part by U.S. Department of Energy, contract DE-FG02-91ER40685
} 


\section{Introduction}

Integrable systems have played an increasingly important role in the study of string theories. In fact, in this connection, it is the supersymmetric integrable systems which become naturally more relevant. Although many properties of the bosonic integrable systems are quite well understood, the same is not true for supersymmetric systems. For example, it is well known that the bosonic integrable systems can be described naturally through Lax operators which are pseudo-differential operators [1]. It is also known through the works of Drinfeld and Sokolov (DS) [2] that given a Lax description of a bosonic integrable system, one can systematically obtain from it a zero curvature formulation of the same system where the matrices (gauge potentials) belong to the symmetry group $s l(n)$ with $n$ related to the order of the pseudo-differential operator. The Drinfeld-Sokolov formalism connecting the scalar Lax formalism to the matrix zero curvature formalism is quite important for it shows that one can uniquely associate with every integrable nonlinear partial differential equation, a unique affine Kac-Moody algebra [2, 3]. The zero curvature formulation depends crucially on the grading of the $\operatorname{sl}(n)$ algebra. While the work of DS uses the principal grading of the underlying algebra, an alternate homogeneous grading leads to models of AKNS type [4, 5]. An essential element in all these algebraic constructions of the integrable hierarchies is the semi-simple (diagonalizable) element $\Lambda$. For the AKNS models, $\Lambda=\lambda E$ is taken to be non-regular element while in the original work of DS, it is assumed to be regular. In the case of the AKNS models, a further simplification occurs in that the construction of the integrable hierarchies can be carried out in a straightforward manner using the symmetric space technique.

In contrast, while the supersymmetric integrable systems are well understood in the Lax formalism with scalar pseudo-differential operators in superspace [6], most of the zero curvature formulations are given in components [0]-11] (see however [12, 13]). The underlying symmetry groups in the zero curvature formulation turn out to be the graded $s l$ or $o s p$ groups as one would expect, but the actual zero curvature formulations are carried out by brute force. In other words, a satisfactory generalization of the DS formalism in the superspace does not yet exist which would systematically associate with a scalar Lax operator in superspace, a zero curvature condition and thereby associate with a set of integrable supersymmetric nonlinear partial differential equation a unique super Kac-Moody algebra.

In this letter, we propose such a generalization for the models of AKNS type. Namely, we work with manifestly supersymmetric $1+1$ dimensional (constrained) KP hierarchies which have superfield formulation with $N$ (Extended) supersymmetry. In section 2, we first describe how one can systematically obtain a zero curvature formulation starting from a scalar Lax operator in superspace. The underlying symmetry algebra naturally corresponds to the graded algebra, $s l(n+1, n)$. We, then, obtain the integrable hierarchies from the superspace zero curvature condition using the method of symmetric space and the nonAbelian gauge technique. In section 3, we work out explicitly the case of $N=1$ super KP hierarchy as an example of the general ideas of section 2 . In section 4 , we work out the $N=2$ example in detail. 


\section{Zero-curvature Equation and Gauge Techniques for the Supersymmetric Constrained Lax Operator}

\subsection{Matrix Eigenvalue Problem for the Supersymmetric Constrained Lax Operator}

We first describe a method of associating a matrix eigenvalue equation to the eigenvalue equation for the pseudo-differential Lax operator $L$ of the grade zero of the following form [14, 15]

$$
\begin{aligned}
L \psi_{B A}\left(t, \theta_{1}, \ldots, \theta_{N}\right) & =\left(\partial+\sum_{a=1}^{m} \sum_{j=1}^{N} \Phi_{a}\left(t, \theta_{1}, \ldots, \theta_{N}\right) D_{\theta_{j}}^{-1} \Psi_{a}\left(t, \theta_{1}, \ldots, \theta_{N}\right)\right) \psi_{B A}\left(t, \theta_{1}, \ldots, \theta_{N}\right) \\
& =\lambda \psi_{B A}\left(t, \theta_{1}, \ldots, \theta_{N}\right)
\end{aligned}
$$

where the superfields $\Phi_{a}\left(t, \theta_{1}, \ldots, \theta_{N}\right)$ and $\Psi_{a}\left(t, \theta_{1}, \ldots, \theta_{N}\right)$ are, respectively, eigenfunctions and adjoint eigenfunctions of $L$, satisfying

$$
\partial_{n} \Phi_{a}=(L)_{+}^{n} \Phi_{a} ; \quad \partial_{n} \Psi_{a}=-\left(L^{*}\right)_{+}^{n} \Psi_{a} ; \quad n=1,2, \ldots
$$

In the sequel we will choose the following grading $\left|\Phi_{a}\right|=0$ and $\left|\Psi_{a}\right|=1$. Moreover, $D_{\theta j}$ are covariant derivatives of the form: $D_{\theta_{j}}=\frac{\partial}{\partial \theta_{j}}+\theta_{j} \partial$, with $\partial f=\partial f / \partial x$. They satisfy $D_{\theta_{j}}^{2}=\partial$ for all $j=1, \ldots, N$. Let $S_{a}\left(t, \theta_{1}, \ldots, \theta_{N}\right)$ be a function such that

$$
\partial S_{a}\left(t, \theta_{1}, \ldots, \theta_{N}\right)=\Psi_{a}\left(t, \theta_{1}, \ldots, \theta_{N}\right) \psi_{B A}\left(t, \theta_{1}, \ldots, \theta_{N}\right)
$$

As described in [16] (see also [17]) $S_{a}$ exists and is defined up to a constant. In terms of the functions in (3) the eigenvalue equation (1) takes a form:

$$
\begin{aligned}
\partial \psi_{B A}\left(t, \theta_{1}, \ldots, \theta_{N}\right) & +\sum_{a=1}^{m} \sum_{j=1}^{N} \Phi_{a}\left(t, \theta_{1}, \ldots, \theta_{N}\right) D_{\theta j} S_{a}\left(t, \theta_{1}, \ldots, \theta_{N}\right) \\
& =\lambda \psi_{B A}\left(t, \theta_{1}, \ldots, \theta_{N}\right)
\end{aligned}
$$

We now associate to the eigenvalue problem a column vector $\chi$ :

$$
\begin{aligned}
& \chi^{T}\left(t, \theta_{1}, \ldots, \theta_{N}\right) \equiv\left(\psi_{B A}, D_{\theta 1} \psi_{B A}, \ldots, D_{\theta N} \psi_{B A}, \ldots, D_{\theta 1} D_{\theta 2} \cdots D_{\theta N} \psi_{B A},\right. \\
& \left.D_{\theta 1} S_{1}, \ldots, D_{\theta 1} S_{m}, D_{\theta 2} S_{1}, \ldots, D_{\theta 2} S_{m}, \ldots, D_{\theta 1} D_{\theta 2} \cdots D_{\theta N} S_{m}\right)
\end{aligned}
$$

where we have not explicitly shown all the ordered terms $D_{\theta j_{1}} \cdots D_{\theta j_{k}}, k \leq N$ such that $1 \leq j_{1}<j_{2}<\ldots<j_{k} \leq N$. In this way $\chi$ contains $1+m\left(2^{N}-1\right)+\left(2^{N}-1\right)=$ $2^{N}+m\left(2^{N}-1\right)$ elements and we find a one to one correspondence between the pseudodifferential Lax eigenvalue equation (1) and the first order matrix equation:

$$
(\partial \cdot I-\lambda E+A) \chi=0
$$

where $E=\operatorname{diag}(\underbrace{1, \ldots, 1}_{2^{N}}, \underbrace{0, \ldots, 0}_{m\left(2^{N}-1\right)}$,$) and A$ is a $\left(2^{N}+m\left(2^{N}-1\right)\right) \times\left(2^{N}+m\left(2^{N}-1\right)\right)$ matrix which depends only on the superfields appearing in $L$. Since both matrices $E$ and $A$ have 
zero super-trace, we notice the appearance of the $s l\left(2^{N-1}+m 2^{N-1}, m\left(2^{N-1}-1\right)+2^{N-1}\right)$ algebraic structure. Especially for the case $m=1$ we find the $\operatorname{sl}(n+1, n)$ structure with $n=2^{N}-1$.

Let us furthermore describe the higher flows in terms of the matrix equation:

$$
\left(\frac{\partial}{\partial t_{n}}+B_{n}\right) \chi=0 \quad ; \quad n=2,3, \ldots
$$

Assuming commutativity of flows $\left[\partial_{x}, \frac{\partial}{\partial t_{n}}\right]=0$ we arrive at the usual zero-curvature (or Zakharov-Shabat) equation:

$$
\partial_{n} A-\partial B_{n}+\lambda\left[E, B_{n}\right]-\left[A, B_{n}\right]=0
$$

This, therefore, provides the generalization of the DS formalism to the superspace.

\subsection{The Gauge Technique of Solving the Zero-Curvature equa- tions}

To establish notation we will now present few facts about the hermitian symmetric spaces.

Given is the algebra $\mathcal{G}$ containing a semi-simple element $E \in \mathcal{G}$, which allows the decomposition $\mathcal{G}=\operatorname{Ker}\left(\operatorname{ad}_{E}\right) \oplus \operatorname{Im}\left(\operatorname{ad}_{E}\right)$ where "ad" represents the adjoint map. Introduce, notation $\mathcal{K} \equiv \operatorname{Ker}\left(\operatorname{ad}_{E}\right)$ and $\mathcal{M} \equiv \operatorname{Im}\left(\operatorname{ad}_{E}\right)$. We work here with $\mathcal{G} / \mathcal{K}$ being a Hermitian symmetric space, i.e. satisfying

$$
[\mathcal{K}, \mathcal{K}] \in \mathcal{K}, \quad[\mathcal{M}, \mathcal{K}] \in \mathcal{M}, \quad[\mathcal{M}, \mathcal{M}] \in \mathcal{K}
$$

as well as

$$
\left.\left(\operatorname{ad}_{E}\right)^{2}\right|_{\mathcal{M}}=I_{\left.\right|_{\mathcal{M}}}
$$

The well-known example of such algebras $\mathcal{G}$ are $\operatorname{sl}(n), n \geq 2$, which underlie the generalized AKNS models [4, 5]. In our discussions, we will be interested in the example of the graded algebras $s l(n+1, n)$ with $n \geq 1$. As we will see later, a specially relevant example is provided by $\operatorname{sl}(2,1)$ with the (super-)traceless semi-simple element:

$$
E=\left(\begin{array}{lll}
1 & 0 & 0 \\
0 & 0 & 0 \\
0 & 0 & 1
\end{array}\right)
$$

In this case, the spaces $\operatorname{Im}\left(\operatorname{ad}_{E}\right)$ and Ker $\left(\operatorname{ad}_{E}\right)$ can be easily checked to have the form

$$
\operatorname{Ker}\left(\operatorname{ad}_{E}\right)=\left(\begin{array}{ccc}
a_{1} & 0 & a_{4} \\
0 & a_{2} & 0 \\
a_{5} & 0 & a_{3}
\end{array}\right) ; \operatorname{Im}\left(\operatorname{ad}_{E}\right)=\left(\begin{array}{ccc}
0 & b_{1} & 0 \\
b_{2} & 0 & f_{1} \\
0 & f_{2} & 0
\end{array}\right)
$$

We now turn our attention to the eigenvalue eq. (6) and work with the general case of

$$
A=A_{\mathcal{K}}+A_{\mathcal{M}} \in \operatorname{Im}\left(\operatorname{ad}_{E}\right)+\operatorname{Ker}\left(\operatorname{ad}_{E}\right)=\mathcal{G}
$$


We will make only one restriction on $A$, namely we will require that the number of independent components of the (adjoint) eigenfunctions in $A$ does not exceed the algebraic dimensionality of $\operatorname{Im}\left(\operatorname{ad}_{E}\right)$. Or in other words we will require that the phase space is spanned by the matrix elements of $A_{\mathcal{M}}$ (namely, $b_{1}, b_{2}, f_{1}, f_{2}$ for the example in eq.(12)) and, consequently, the matrix elements of $A_{\mathcal{K}}$ depend functionally on those of $A_{\mathcal{M}}$.

We now notice that the gauge potential in the direction of $\mathcal{K}$ can be completely gauged away so that the eigenvalue eq. (6) can be rewritten as

$$
(\partial \cdot I-\lambda E+\mathcal{A}) \bar{\chi}=0
$$

with

$$
\begin{aligned}
\mathcal{A} & \equiv G^{-1} A G+G^{-1} \partial G=G^{-1} A_{\mathcal{M}} G \\
\bar{\chi} & \equiv G^{-1} \chi
\end{aligned}
$$

where $G$ is determined from the condition:

$$
G^{-1} A_{\mathcal{K}} G+G^{-1} \partial G=0 \quad ; \quad G=\exp \left(-\int^{x} A_{\mathcal{K}} d x\right)
$$

Because of condition (17) it holds that the matrix $\mathcal{A}$ belongs to the image of $\operatorname{ad}_{E}$ [5]:

$$
\mathcal{A} \in \operatorname{Im}\left(\operatorname{ad}_{E}\right) \text {. }
$$

We turn our attention back to the zero-curvature eq.(8) derived from the eigenvalue problem (14) and containing $A$ substituted by $\mathcal{A}$ as in (18). We note that the gauge transformation was chosen to rotate the $\mathcal{K}$ component of $A$ from the Lax equation, but, in general, this transformation would lead to both the components in $B$ and, therefore, we split the $B_{n}$ matrix into its Ker $\left(\operatorname{ad}_{E}\right)$ and $\operatorname{Im}\left(\operatorname{ad}_{E}\right)$ components

$$
B_{n}=B_{n}^{\mathcal{K}}+B_{n}^{\mathcal{M}}
$$

Accordingly, the zero-curvature eq.(\$) splits into

$$
\begin{aligned}
\partial B_{n}^{\mathcal{K}}+\left[\mathcal{A}, B_{n}^{\mathcal{M}}\right] & =0 \\
\partial_{n} \mathcal{A}-\partial B_{n}^{\mathcal{M}}+\lambda\left[E, B_{n}^{\mathcal{M}}\right]-\left[\mathcal{A}, B_{n}^{\mathcal{K}}\right] & =0
\end{aligned}
$$

along the Ker $\left(\operatorname{ad}_{E}\right)$ and $\operatorname{Im}\left(\operatorname{ad}_{E}\right)$ directions. Equation (20) can readily be integrated to yield

$$
B_{n}^{\mathcal{K}}=-\partial^{-1}\left(\left[\mathcal{A}, B_{n}^{\mathcal{M}}\right]\right)-\lambda^{n} E
$$

where the last term is an "integration constant" chosen to ensure agreement with the limiting case $n=1$ for which $B_{1}=\mathcal{A}-\lambda E$. Plugging eq.(22) into eq.(21) we get

$$
\partial_{n} \mathcal{A}-\partial B_{n}^{\mathcal{M}}+\lambda\left[E, B_{n}^{\mathcal{M}}\right]+\left[\mathcal{A}, \partial^{-1}\left(\left[\mathcal{A}, B_{n}^{\mathcal{M}}\right]\right)\right]-\lambda^{n}[E, \mathcal{A}]=0
$$

We search for solutions of (23) of the form

$$
B_{n}^{\mathcal{M}}=\sum_{i=0}^{n-1} \lambda^{i} B_{n}^{\mathcal{M}}(i)
$$


From (23) we obtain for coefficients of $\lambda^{n}$ :

$$
B_{n}^{\mathcal{M}}(n-1)=\mathcal{A}
$$

after use of relation (10). We also find from (23) the recursion relation

$$
B_{n}^{\mathcal{M}}(i-1)=\mathcal{R} B_{n}^{\mathcal{M}}(i)
$$

for coefficients of expansion of $B_{n}^{\mathcal{M}}$ with the recursion matrix given by

$$
\mathcal{R} \equiv a d_{E}\left(\partial-a d_{\mathcal{A}} \partial^{-1} a d_{\mathcal{A}}\right)
$$

As a consequence we find

$$
\operatorname{ad}_{E}\left(\partial_{n} \mathcal{A}\right)=\mathcal{R}\left(B_{n}^{\mathcal{M}}(0)\right)=\mathcal{R}^{n}(\mathcal{A}) \quad ; \quad \partial_{n} \mathcal{A}=\operatorname{ad}_{E} \mathcal{R} a d_{E}\left(\partial_{n-1} \mathcal{A}\right)
$$

Hence, in principle, once the recursion operator $\mathcal{R}$ is determined, we can find all the evolution equations of the hierarchy. Let us consider, for example, the first non-trivial case of $n=2$ for which we find:

$$
\operatorname{ad}_{E}\left(\partial_{2} \mathcal{A}\right)=\mathcal{R}^{2}(\mathcal{A})=\partial_{x}^{2} \mathcal{A}-\frac{1}{2} \operatorname{ad}_{E} \operatorname{ad}_{\mathcal{A}} \operatorname{ad}_{\mathcal{A}} \operatorname{ad}_{E}(\mathcal{A})
$$

From first of eqs. 28) we easily find

$$
\operatorname{ad}_{E}\left(\partial_{n-1} \mathcal{A}\right)=B_{n}^{\mathcal{M}}(0) \rightarrow \partial B_{n}^{\mathcal{K}}(0)=-\operatorname{ad}_{\mathcal{A}} \operatorname{ad}_{E}\left(\partial_{n-1} \mathcal{A}\right)
$$

where use was made of eq.(22). Repeating arguments of [5] and [3] we find that

$$
\begin{aligned}
\partial_{n} \operatorname{Tr}\left(\mathcal{A}^{2}\right) & =2 \operatorname{Tr}\left([E,[E, \mathcal{A}]] \partial_{n} \mathcal{A}\right)=-2 \operatorname{Tr}\left(\operatorname{Ead}_{\mathcal{A}} \operatorname{ad}_{E}\left(\partial_{n} \mathcal{A}\right)\right) \\
& =2 \partial \operatorname{Tr}\left(E B_{n+1}^{\mathcal{K}}(0)\right)=2 \partial \operatorname{Tr}\left(E B_{n+1}(0)\right)
\end{aligned}
$$

Assuming now that $\mathcal{H}_{1}=\frac{1}{2} \operatorname{Tr}\left(\mathcal{A}^{2}\right)$ defines the first Hamiltonian density, it follows that $\mathcal{H}_{n}=\operatorname{Tr}\left(E B_{n+1}(0)\right)$ satisfies the basic relation $\partial \mathcal{H}_{n}=\partial_{n} \mathcal{H}_{1}$. Note also, that $\operatorname{Tr}\left(\mathcal{A}^{2}\right)=$ $\operatorname{Tr}\left(A_{\mathcal{M}}^{2}\right)$. This shows, in general, how we can use the methods of symmetric space as well as the gauge technique to obtain the hierarchy equations from the zero curvature condition. In

the next two sections, we will work out explicitly examples corresponding to the $N=1$ and $N=2$ cases.

\section{Supersymmetric AKNS, Matrix Formulation and Gaug- ing Technique}

The Lax operator of the supersymmetric AKNS model can be written as [14:

$$
L_{A K N S}=\partial+\Phi D_{\theta}^{-1} \Psi .
$$


According, to the discussion above introduce $S$ such that $\partial_{x} S=\Psi \psi_{B A}$, and choose the independent variables $\psi_{B A}, D_{\theta}(S)$ and $D_{\theta}\left(\psi_{B A}\right)$. Then eq. (6) is equivalent with

$$
\left(\begin{array}{ccc}
\partial-\lambda & \Phi & 0 \\
-D_{\theta}(\Psi) & \partial & \Psi \\
\Phi \Psi & D_{\theta} \Phi & \partial-\lambda
\end{array}\right)\left(\begin{array}{c}
\psi_{B A} \\
D_{\theta}(S) \\
D_{\theta}\left(\psi_{B A}\right)
\end{array}\right)=0
$$

If we use the matrix $E$ as in (11) and denote by

$$
A=\left(\begin{array}{ccc}
0 & \Phi & 0 \\
-D_{\theta}(\Psi) & 0 & \Psi \\
\Phi \Psi & D_{\theta}(\Phi) & 0
\end{array}\right) \quad ; \quad \chi=\left(\begin{array}{c}
\psi_{B A} \\
D_{\theta}(S) \\
D_{\theta}\left(\psi_{B A}\right)
\end{array}\right)
$$

then eq. (33) reads $(\partial-\lambda E+A) \chi=0$ The matrices (34) suggest an underlying sl $(2,1)$ algebraic structure. We employ the gauge technique to bring the matrix $A$ from eq.(34) into the form of $\operatorname{Im}\left(\operatorname{ad}_{E}\right)$ as in eq.(12). To do it, we apply the gauge transformation from (15) with $G$ as in (17). In this case it is given by $G=\exp \left(-\int^{x} \Phi \Psi \kappa_{1} d x\right)$ with

$$
\kappa_{1}=\left(\begin{array}{ccc}
0 & 0 & 0 \\
0 & 0 & 0 \\
1 & 0 & 0
\end{array}\right)
$$

After the gauge transformation performed as in eq.(15) we obtain:

$$
\mathcal{A}=\left(\begin{array}{ccc}
0 & \Phi & 0 \\
-\left(D_{\theta} \Psi\right)+\left(D_{\theta}{ }^{-2} \Phi \Psi\right) \Psi & 0 & \Psi \\
0 & \left(D_{\theta} \Phi\right)+\left(D_{\theta}{ }^{-2} \Phi \Psi\right) \Phi & 0
\end{array}\right)
$$

which can be plugged directly into eq.(29) to obtain immediately the $n=2$ evolution equation of the hierarchy for $\Phi, \Psi$ :

$$
\begin{aligned}
& \partial_{2} \Phi=\Phi^{\prime \prime}-2\left(\Phi \Psi\left(D_{\theta} \Phi\right)-\left(D_{\theta} \Psi\right) \Phi^{2}\right) \\
& \partial_{2} \Psi=-\Psi^{\prime \prime}-2\left(D_{\theta} \Psi\right) \Phi \Psi
\end{aligned}
$$

These are, indeed, the equations of the hierarchy. Note, that according to the discussion following eq.(31) the first two Hamiltonian densities are

$$
\begin{aligned}
& \mathcal{H}_{1}=\frac{1}{2} \operatorname{Tr}\left(A^{2}\right)=\Phi \bar{\Psi}-\bar{\Phi} \Psi=-\left(\Phi\left(D_{\theta} \Psi\right)+\left(D_{\theta} \Phi\right) \Psi\right) \\
& \mathcal{H}_{2}=\bar{\Psi} \Phi^{\prime}-\Phi \bar{\Psi}^{\prime}+\Psi \bar{\Phi}^{\prime}-\Psi^{\prime} \bar{\Phi}
\end{aligned}
$$

where $\bar{\Phi} \equiv\left(D_{\theta} \Phi\right)+\left(D_{\theta}{ }^{-2} \Phi \Psi\right) \Phi$ and $\bar{\Psi} \equiv-\left(D_{\theta} \Psi\right)+\left(D_{\theta}{ }^{-2} \Phi \Psi\right) \Psi$. The corresponding Poisson bracket structures for the "canonical" variables $\Phi, \bar{\Psi}, \Psi, \bar{\Phi}$ are of the form:

$$
P_{1}=\left(\begin{array}{cccc}
0 & -1 & 0 & 0 \\
1 & 0 & 0 & 0 \\
0 & 0 & 0 & -1 \\
0 & 0 & -1 & 0
\end{array}\right)
$$


and $P_{2}$ given by

$$
\left(\begin{array}{cccc}
-2 \Phi \partial^{-1} \Phi & -\partial-\bar{\Phi} \partial^{-1} \Psi+2 \Phi \partial^{-1} \bar{\Psi} & \Phi \partial^{-1} \Psi & -\bar{\Phi} \partial^{-1} \Phi-\Phi \partial^{-1} \bar{\Phi} \\
-\partial+\Psi \partial^{-1} \bar{\Phi}+2 \bar{\Psi} \partial^{-1} \Phi & -2 \bar{\Psi} \partial^{-1} \bar{\Psi} & -\bar{\Psi} \partial^{-1} \Psi-\Psi \partial^{-1} \bar{\Psi} & \bar{\Psi} \partial^{-1} \bar{\Phi} \\
\Psi \partial^{-1} \Phi & -\Psi \partial^{-1} \bar{\Psi}-\bar{\Psi} \partial^{-1} \Psi & 0 & \partial-\bar{\Psi} \partial^{-1} \Phi \\
-\bar{\Phi} \partial^{-1} \Phi-\Phi \partial^{-1} \bar{\Phi} & \bar{\Phi} \partial^{-1} \bar{\Psi} & -\partial+\Phi \partial^{-1} \bar{\Psi} & 0
\end{array}\right)
$$

where the matrix elements should be identified as $P_{\{i j\}} \delta(x-y)=\left\{X_{i}(x), X_{j}(y)\right\}$ for $X=\Phi, \bar{\Psi}, \Psi, \bar{\Phi}$. This bracket structures are consistent with expression $\mathcal{R}=P_{2} P_{1}^{-1}$ for the recursion matrix $\mathcal{R}$ such that $\partial_{n} X=\mathcal{R} \partial_{n-1} X$ They also lead to the correct equations of motion (37)-(38) via $\partial_{2} X=\left\{H_{2}, X\right\}_{2}$ with $H_{2}=\frac{1}{2} \int d x \mathcal{H}_{2}$. Note, that it also holds that $\partial_{1} X=\left\{H_{2}, X\right\}_{1}=\left\{H_{1}, X\right\}_{2}$ with $H_{1}=\int d x \mathcal{H}_{1}$. As explained in 18 presence of the non-local terms $\partial_{x}^{-1} \delta(x-y)$ in (42) requires special care when verifying the Jacobi identities for the bracket $P_{2}$. We postpone dealing with this problem for a later publication.

\section{$4 \quad N=2$ supersymmetric Lax}

In this section we illustrate our general formalism with an example possessing $N=2$ supersymmetry. Consider, namely the superspace described in terms of $\left(t, \theta_{1}, \theta_{2}\right)$, and the Lax operator:

$$
L=\partial+\Phi\left(t, \theta_{1}, \theta_{2}\right) D_{\theta_{1}}^{-1} \Psi\left(t, \theta_{1}, \theta_{2}\right)+\Phi\left(t, \theta_{1}, \theta_{2}\right) D_{\theta_{2}}^{-1} \Psi\left(t, \theta_{1}, \theta_{2}\right) .
$$

In order to obtain the hierarchy equations via the symmetric algebra formalism we will associate to the eigenvalue equation

$$
L \psi_{B A}\left(t, \theta_{1}, \theta_{2}\right)=\lambda \psi_{B A}\left(t, \theta_{1}, \theta_{2}\right)
$$

a matrix eigenvalue equation $(\partial \cdot I-\lambda E+A) \chi=0$ with

$$
E=\operatorname{diag}(1,1,0,0,0,1,1)
$$

and

$$
\chi^{T}=\left(\psi_{B A}, D_{\theta 1} D_{\theta 2} \psi_{B A}, D_{\theta 1} S, D_{\theta 2} S, D_{\theta 1} D_{\theta 2} S, D_{\theta 1} \psi_{B A}, D_{\theta 2} \psi_{B A}\right)
$$

We find

$$
A=\left(\begin{array}{ccccccc}
0 & 0 & \Phi & \Phi & 0 & 0 & 0 \\
\left(D_{1}-D_{2}\right) \Phi \Psi & 0 & D_{12} \Phi & D_{12} \Phi & -\left(D_{1}+D_{2}\right) \Phi & -\Phi \Psi & \Phi \Psi \\
-D_{1} \Psi & 0 & 0 & 0 & 0 & \Psi & 0 \\
-D_{2} \psi & 0 & 0 & 0 & 0 & 0 & \Psi \\
-D_{12} \Psi & -\Psi & 0 & 0 & 0 & -D_{2} \Psi & D_{1} \Psi \\
\Phi \Psi & 0 & D_{1} \Phi & D_{1} \Phi & \Phi & 0 & 0 \\
\Phi \Psi & 0 & D_{2} \Phi & D_{2} \Phi & -\Phi & 0 & 0
\end{array}\right)
$$

where for brevity we used $D_{i}=D_{\theta i}, i=1,2$ and $D_{12}=D_{\theta 1} D_{\theta 2}$.

Applying the gauge technique within the setting of $\operatorname{sl}(4,3)$ algebra sends $A \rightarrow \mathcal{A} \in$ Im $\left(\operatorname{ad}_{E}\right)$ and results in the $N=2$ AKNS hierarchy evolution equations:

$$
\begin{aligned}
& \partial_{2} \Phi=\Phi^{\prime \prime}-2\left(\Phi \Psi\left(D_{\theta 1}+D_{\theta 2}\right) \Phi-\Phi^{2}\left(D_{\theta 1}+D_{\theta 2}\right) \Psi\right) \\
& \partial_{2} \Psi=-\Psi^{\prime \prime}-2 \Phi \Psi\left(D_{\theta 1}+D_{\theta 2}\right) \Psi
\end{aligned}
$$




\section{Conclusion}

In this letter, we have generalized the Drinfeld-Sokolov formalism to superspace which allows us to systematically construct a zero curvature condition associated with a supersymmetric integrable system described by a scalar Lax operator in superspace. We have shown, in general, how the AKNS type of models in superspace can be obtained from the zero curvature condition through the method of symmetric space and gauge technique. We have illustrated the formalism by explicitly working out two examples with $N=1$ and $N=2$ supersymmetry.

\section{References}

[1] I.M. Gelfand and L.A. Dickey, Functional Analysis and Its Application 10 (1976) 4

[2] V. G. Drinfeld and V. V. Sokolov, J. Soviet Math. 30 (1985) 1975; Soviet. Math. Dokl. 23 (1981) 457 ;

[3] G. Wilson, Ergod. Th. and Dynam. Sys. 1 (1981) 361

[4] A.P. Fordy and P.P. Kulish, Commun. Math. Phys. 89 (1983) 427; A.P. Fordy, in Soliton Theory: a Survey of Results, (ed. A.P. Fordy) University Press, Manchester (1990), pg. 315

[5] H. Aratyn, J.F. Gomes and A.H. Zimerman, J. Math. Phys. 36 (1995) 3419 (hep-th/9408104)

[6] B. Kupershmidt, "Elements of Superintegrable Systems: basic techniques and results", Kluwer Acad. Publ., 1987

[7] M. Gürses and Ö. Ŏguz, Phys. Lett. 108A (1985) 437

[8] A. Das and S. Roy, J. Math. Phys. 31 (1990) 2145; A. Das, W. -J. Huang and S. Roy, Int. J. Mod. Phys. A7 (1992) 3447

[9] A. Das, W. -J. Huang and S. Roy, Int. J. Mod. Phys. A7 (1992) 4293

[10] J. C. Brunelli and A. Das, Phys. Lett. 337B (1994) 303 (hep-th/9406214); Phys. Lett. 354B (1995) 307 (hep-th/9504030); Int. J. Mod. Phys. A10 (1995) 4563 (hep-th/9505093)

[11] A. Das and S. Roy, Mod. Phys. Lett. A11 (1996) 1317 (hep-th/9511091)

[12] T. Inami and H. Kanno, Commun. Math. Phys. 136 (1991) 519

[13] F. Toppan, Int. J. Mod. Phys. A11 (1996) 3257 (hep-th/9506133)

[14] H. Aratyn and C. Rasinariu, Phys. Lett. 391B (1997) 99 (hep-th/9608107)

[15] Z. Popowicz, J. Physics A29 (1996) 1281 (hep-th/9510185)

[16] H. Aratyn, E. Nissimov and S. Pacheva, Method of Squared Eigenfunction Potentials in Integrable Hierarchies of KP Type, solv-int/9701017

[17] Y. Cheng, J. Math. Phys. 33 (1992) 3774

[18] H.S. Blas Achic, L.A. Ferreira, J.F. Gomes and A.H. Zimerman, Some comments on the bi(tri)-Hamiltonian structure, solv-int/9701012 